\title{
Supplementing Milk Replacer with Omega-3 Fatty Acids from Fish Oil on Immunocompetence and Health of Jersey Calves
}

\author{
M. A. Ballou ${ }^{1}$ and E. J. DePeters ${ }^{2}$ \\ Department of Animal Science and Nutritional Biology Graduate Group, University of California, Davis, 95616
}

\begin{abstract}
Fifty-one Jersey bull calves ( $5 \pm 1 \mathrm{~d}$ old) were assigned to 1 of 3 milk replacers to determine the effects of increasing doses of n-3 fatty acids from fish oil on immunocompetence and health. All calves were fed a $22.5 \%$ crude protein and $18 \%$ lipid industry standard milk replacer supplemented with an additional $2 \%$ fatty acids. The 3 treatments differed only in the supplemental lipid source and included a 3:1 mix of corn and canola oils; a 1:1 blend of fish oil and the 3:1 mix of corn and canola oils; and fish oil only. All treatments were supplemented with $150 \mathrm{mg}$ of vitamin $\mathrm{E} / \mathrm{kg}$ of milk replacer. Body weight, height at withers, and length between withers and pins were measured weekly. Fecal and respiratory scores were recorded multiple times daily, and peripheral blood samples were collected on d $0,7,14,21$, and 42 for hematologic and metabolic analyses. Immunocompetence of calves was evaluated in vitro by the ability of neutrophils and monocytes to phagocytose Escherichia coli and produce an oxidative burst and in vivo as the change in ear thickness after an intradermal injection of phytohemagglutinin-P, and the primary and secondary humoral responses to ovalbumin. Production and health parameters were unaffected by treatments. There were no significant treatment or treatment $\times$ time effects on phagocytosis; however, there was a significant quadratic response for the percentage of neutrophils producing an oxidative burst. Fish oil did not affect the change in ear thickness in response to phytohemagglutinin-P. There was also no treatment effect on the primary IgG humoral response to ovalbumin, but there was a significant quadratic treatment effect on the secondary IgG response. Adding fish oil to milk replacer altered various immune responses, and the effect was dose-dependent; however, neither production performance nor indices
\end{abstract}

Received January 15, 2008.

Accepted May 28, 2008.

${ }^{1}$ Current address: Department of Animal and Food Sciences, Texas Tech University, Lubbock, TX 79409.

${ }^{2}$ Corresponding author: ejdepeters@ucdavis.edu of health were altered when fish oil replaced 5 to $10 \%$ of the fatty acids in milk replacer.

Key words: calf, fish oil, health, immune system

\section{INTRODUCTION}

It is well recognized that calves are highly susceptible to microorganisms and subsequently death, a response that may reflect deficiencies in both innate and adaptive immune responses (Roy, 1990). The high mortality rate of calves was due to the high incidence and poor survival of septicemic calves (Lofstedt et al., 1999). Strategies need to be identified that either limit the incidence of septicemic calves or modify the pathophysiological response of the septicemic calf to lessen the high mortality associated with the acute response. Data presented in a companion paper (Ballou et al., 2008) indicated that supplementing a milk replacer with fish oil (FO) attenuated many aspects of the acute phase response. This response was linear in the range of 5 to $10 \%$ of the lipid in milk replacer replaced as fatty acids (FA) from FO. This suggests a benefit to calf health, possibly providing a necessary balance between an appropriate versus an excessive acute phase response during systemic infection. However, the effect of supplementing FO at these levels on other functional immune responses and overall health in calves has not been previously investigated.

In addition to attenuating the acute phase response, increasing dietary FO modulated other aspects of immunocompetence in rodents and humans (Calder, 2006). Experimental evidence supports a suppressed cell-mediated immune response when supplemented with high doses of FO (Meydani et al., 1991); however, at more moderate doses of FO, cell-mediated immunity was unaffected (Yaqoob et al., 2000). Conflicting findings regarding the effects of supplemental FO on the development of humoral response were reported (Virella et al., 1991; Hall et al., 2003). In addition to effects of $\mathrm{FO}$ on lymphocyte functions, aspects of innate immunity, including phagocytosis and oxidative burst, are influenced by supplemental FO. Early research suggested that the degree of unsaturation of the FA in macrophage membranes affected their ability to phago- 
cytose pathogens. Subsequently, Calder et al. (1990) confirmed that the rate of phagocytosis was positively correlated with the degree of FA unsaturation; however, they also noted that macrophages enriched with the n-3 FA, eicosapentaenoic acid (C20:5; EPA) and docosahexaenoic acid (C22.6; DHA), had lower phagocytic activity than would be expected based on their degree of unsaturation. Supplementing a high dose of FO decreased neutrophil oxidative burst by approximately 30\%; however, a lower dose of FO had no influence on superoxide generation (D'Ambola et al., 1991). In contrast, a significant negative correlation was observed between the oxidative burst of neutrophils and the n-6:n-3 FA ratio of the neutrophil membrane (Kew et al., 2003). Taken together, these data suggest that the effects of FO on immunocompetence are dosedependent. Therefore, the objectives of this study were to determine the effects of increasing doses of n-3 FA from $\mathrm{FO}$ on aspects of functional immunocompetence and health of calves.

\section{MATERIALS AND METHODS}

\section{Experimental Design, Calves, and Diets}

Fifty-one Jersey bull calves ( $5 \pm 1 \mathrm{~d}$ old) were completely randomized to 1 of 3 treatment diets, differing only in the FA composition of the milk replacer. To assure adequate randomization, BW and total serum protein were evaluated after assignment of treatments. The base diet given to all calves was a $22.5 \% \mathrm{CP}$ and $18 \%$ lipid industry standard milk replacer (Calva Products, Acampo, CA). The treatments were applied by supplementing the base diet with an additional $2 \% \mathrm{FA}$. Treatments included a 3:1 mix of corn and canola oils (control), a 1:1 mix of FO (Omega Proteins, Houston, TX) and the control oil (blend diet), and FO only (fish diet). Predicted average daily gains for wk 1 and 2,3 to 5 , and 6 to 8 were 200,400, and $600 \mathrm{~g} / \mathrm{d}$, respectively. All calves were supplemented with $150 \mathrm{mg}$ of vitamin $\mathrm{E} / \mathrm{kg}$ of milk replacer, which is approximately 3 times the minimum recommended dose published by the NRC (2001) to prevent nutritional myopathy from lipid peroxidation. No calf starter was offered throughout the experiment, and calves were offered fresh water ad libitum daily. The study was conducted from May 2005 to July 2005. The average temperatures during this time ranged from 18 to $23^{\circ} \mathrm{C}$, with average daily highs ranging from 26 to $35^{\circ} \mathrm{C}$ and lows from 9 to $13^{\circ} \mathrm{C}$.

Calf performance was evaluated once weekly, and measurements included BW, height at the withers, and length between the withers and pins. Calf health was monitored daily. Diarrhea was treated primarily with acidified oral electrolytes (Bluelite C, Tech Mix,
Stewart, MN) added directly to the milk formula. Milk formula was never withheld from calves with diarrhea. Intravenous fluid, $13 \mathrm{~g}$ of $\mathrm{NaHCO}_{3}$ (American Regent, Shirley, NY) added to $1 \mathrm{~L}$ of $5 \%$ dextrose (Baxter, Deerfield, IL), was administered as a bolus to support severely dehydrated and recumbent calves as needed. Indices of health were subjectively recorded multiple times daily by 1 observer under the following guidelines: fecal scores: 1 = firm, well-formed (not hard); 2 $=$ soft, pudding-like; 3 = runny, pancake batter; and 4 = liquid, splatters, pulpy orange juice; Respiratory scores: $1=$ normal; $2=$ runny nose; $3=$ heavy breathing; 4 = cough-moist; 5 = cough-dry; and $6=$ fever (Larson et al., 1977). Other health disorders were treated per veterinary instruction. Samples of milk formula were collected every Monday, Wednesday, and Friday a.m. and composited by week for proximate analyses and FA determination. All animal care was reviewed and approved by the Animal Care and Use Committee of the University of California, Davis.

\section{Blood Collection, Serum Hematologic, and Metabolic Analyses}

Peripheral blood $(20 \mathrm{~mL})$ was collected from the jugular vein into evacuated tubes containing either no additive or $\mathrm{K}_{2}$ EDTA from each calf on d $0,7,14,21$, and 42 relative to the initiation of treatments. White blood cell counts were determined manually using a Unopette (Becton Dickinson, Franklin Lakes, NJ) to dilute the leukocytes and lyse the erythrocytes. Direct measurement of the hematocrit was performed by centrifugation. Total plasma protein was determined using a temperature-controlled refractometer. Serum glucose, BUN, NEFA, triacylglycerol (TG), insulin, and leptin concentrations were analyzed as outlined in Ballou et al. (2008).

\section{Flow Cytometric Whole-Blood Phagocytic and Oxidative Burst Assays}

Peripheral blood was collected into heparinized evacuated tubes from each calf on d 21 after the initiation of treatments. The phagocytosis assay used whole blood stimulated with fluorescent Escherichia coli using a commercial kit (Phagotest, Orpegen Pharma, Heidelberg, Germany) and subsequently analyzed by flow cytometry to analyze the polymorphonuclear leukocyte (PMN) and monocyte populations from 1 sample, independently. The assay was run according to the procedures described by Kampen et al. (2004) with modifications. Briefly, $50 \mu \mathrm{L}$ of heparinized whole blood was incubated in a water bath at $38.5^{\circ} \mathrm{C}$ with $4 \mathrm{\mu L}$ of preopsonized, fluorescein isothiocyanate (FITC)-labeled E. coli; the bacteria:PMN ratio was ap- 
proximately 25:1. Negative control tubes remained on ice throughout the incubation period. After either 2- or 15-min incubations, the samples were removed from the water bath, immediately submerged in an ice water bath, and $50 \mu \mathrm{L}$ of ice-cold quenching solution was added to each tube to quench any extracellular fluorescence. The cells were washed and the erythrocytes lysed and removed. The remaining leukocytes were fixed and stained with propidium iodide. Two- and 15-min incubations were chosen based on preliminary data (not shown) using the approximate 25:1 bacteria:PMN ratio. The data indicated that both the percentage of PMN and monocytes phagocytizing the preopsonized $E$. coli were in the logarithm and stationary phases at 2 and $15 \mathrm{~min}$, respectively.

Oxidative burst was measured using a commercial kit (Bursttest, Orpegen Pharma) according to the modified procedures described by Kampen et al. (2004) except preopsonized E. coli was used. Briefly, $50 \mu \mathrm{L}$ of heparinized whole blood was incubated in a water bath at $38.5^{\circ} \mathrm{C}$ with $10 \mu \mathrm{L}$ of preopsonized Escherichia coli; the bacteria:PMN ratio was approximately 60:1. Negative control tubes remained on ice throughout the incubation period. After $10 \mathrm{~min}$, each sample was removed from the water bath and immediately submerged in an ice water bath for $10 \mathrm{~min}$. Then, $10 \mu \mathrm{L}$ of dihydrorhodamine 123 was added, and each tube was incubated at $38.5^{\circ} \mathrm{C}$ for $10 \mathrm{~min}$, after which each tube was immediately submerged in an ice water bath. The erythrocytes were lysed and removed. The remaining leukocytes were washed, fixed, and stained with propidium iodide. The 60:1 approximate ratio of bacteria to PMN and 10-min incubation were chosen based on preliminary data (not shown). Increasing the bacteria:PMN ratio did not further increase either the percentage or the mean fluorescence intensity of the PMN undergoing an oxidative burst. Extending the incubation time did not further increase the percentage of PMN undergoing an oxidative burst. The oxidative burst capacity of monocytes was negligible using the strain of $E$. coli, which was reported previously (Kampen et al., 2004).

Both the phagocytosis and oxidative burst cell suspensions were measured using a flow cytometer equipped with a $15-\mathrm{mW}, 488-\mathrm{nm}$ air-cooled argon-ion laser (FACS Calibur, Becton Dickinson). During data acquisition, the FL-2 channel was used to set a gate, so that only nucleated cells would be recorded. A conservative, active gate was created around the PMN population, using the forward and side scatter characteristics of the cells. Five thousand PMN events were recorded per sample. Data were analyzed using FlowJo software (Tree Star Inc., Ashland, OR); electronic gates were placed around both the PMN and monocyte populations using the forward and side scatter characteristics of the cells. The percentages of PMN and monocyte cells that were positive for phagocytosis and the mean channel fluorescence of the FL-1 channel were analyzed. The percentage of PMN positive for an oxidative burst and the increase in the geometric mean of the FL- 1 channel for all cells within the PMN gate were used to analyze the oxidative burst assay.

\section{Humoral Response to Ovalbumin and Quil A}

On d 23 and 37 after the initiation of treatments, all calves were injected intramuscularly in the rump with $0.5 \mathrm{mg}$ of ovalbumin (OVA) and $0.5 \mathrm{mg}$ of the adjuvant Quil A in $1 \mathrm{~mL}$ of sterile PBS (Mallard et al., 1997). Sterility of the injections was verified by plating $75 \mu \mathrm{L}$ of the working solution on blood agar. Peripheral blood was collected on $d 23,30,33,37,44$, and 48 after the initiation of treatments to determine the primary and secondary OVA-specific antibody responses. The IgM and IgG antibodies specific for OVA were measured using an indirect ELISA outlined by Mallard et al. (1997). Briefly, 96-well, flat-bottom Reacti-Bind (Pierce Biotechnology, Rockford, IL) plates were coated with $100 \mu \mathrm{L}(1.4 \mu \mathrm{g}$ of OVA $/ \mu \mathrm{L}$ of carbonate bicarbonate buffer; $\mathrm{pH}=9.4)$ at $4^{\circ} \mathrm{C}$ for $48 \mathrm{~h}$. Plates were then washed 4 times with PBS containing $0.05 \%$ Tween 20 (SigmaAldrich, St. Louis, MO). Plates were blocked with 300 $\mu \mathrm{L}$ of $4 \%$ BSA (Celliance Corp, Norcross, GA) and 5\% sucrose in PBS, $\mathrm{pH}=7.4$, for $2 \mathrm{~h}$. Plates were then washed with PBS-Tween. Serum was diluted 1:50 and 1:200 in wash buffer, and $100 \mu \mathrm{L}$ was added to each well in duplicate. Plates were incubated with serum for $2 \mathrm{~h}$ and then washed with PBS-Tween and $100 \mu \mathrm{L}$ of rabbit anti-bovine IgM (KPL, Gaithersburg, MD) conjugated to alkaline phosphatase, or rabbit anti-bovine IgG (KPL) conjugated to alkaline phosphatase in Trisbuffered saline was added and incubated for $1 \mathrm{~h}$. Plates were washed with PBS-Tween, and $80 \mu \mathrm{L}$ of substrate (10 mg of $p$-nitrophenylphosphate in $10 \mathrm{~mL}$ of glycine buffer; $\mathrm{pH}=10.4$ ) was added. Plates were incubated for exactly $30 \mathrm{~min}$. Absorbance was read at 540 and 410 nm using a VERSA Max microplate reader (Molecular Devices, Sunnyvale, CA), and results were reported as the difference between the 2 absorbances for each serum dilution. Positive controls were from a pool of 6 adult, nonlactating cows taken $7 \mathrm{~d}$ after the second vaccination with OVA + Quil A. On each microplate, the positive control was adjusted to an optical density of 1.0, and the unknowns on each plate were calculated by multiplying the optical density of the positive control/1.0. The interplate CV were 7.3 and $6.9 \%$ for $1: 200$ and 1:50 serum dilutions, respectively. The intraplate CV were 1.4 and $2.5 \%$ for the 1:200 and 1:50 serum dilutions, respectively. 
Table 1. Chemical composition of treatment milk replacers ${ }^{1}$ (least squares means)

\begin{tabular}{|c|c|c|c|c|}
\hline \multirow[b]{2}{*}{ Item } & \multicolumn{3}{|c|}{ Treatment $^{2}$} & \multirow[b]{2}{*}{$\mathrm{SEM}^{3}$} \\
\hline & Control & Blend & Fish & \\
\hline Dry matter, \% & 95.4 & 95.4 & 95.4 & 0.1 \\
\hline Ash, \% DM & 7.97 & 8.14 & 8.03 & 0.15 \\
\hline Crude protein, \% DM & 21.6 & 21.7 & 21.8 & 0.3 \\
\hline Fatty acid, ${ }^{4} \% \mathrm{DM}$ & 19.4 & 19.6 & 19.7 & 0.2 \\
\hline Arachidonic acid, g/100 g of fatty acid & 0.222 & 0.262 & 0.309 & 0.009 \\
\hline Eicosapentaenoic acid, g/100 g of fatty acid & 0.010 & 0.645 & 1.267 & 0.078 \\
\hline Docosahexaenoic acid, g/100 g of fatty acid & 0.010 & 0.560 & 1.103 & 0.066 \\
\hline$n-6, g / 100 \mathrm{~g}$ of fatty acid ${ }^{5}$ & 15.6 & 13.7 & 11.4 & 0.1 \\
\hline $\mathrm{n}-3, \mathrm{~g} / 100 \mathrm{~g}$ of fatty $\mathrm{acid}^{6}$ & 1.5 & 2.8 & 4.1 & 0.2 \\
\hline$n-6: n-3$ & 10.1 & 4.8 & 2.9 & 0.1 \\
\hline
\end{tabular}

${ }^{1}$ Source of milk replacer was Calva Products (Acampo, CA).

${ }^{2}$ Control $=3: 1$ mix of corn and canola oils; Blend $=1: 1$ mix of fish oil and control oil; Fish $=$ fish oil only.

${ }^{3} \mathrm{SEM}$ was determined from analysis of weekly composite samples.

${ }^{4}$ Total fatty acids were determined by gas chromatography.

${ }^{5} \mathrm{n}-6=\mathrm{C} 18: 2, \mathrm{C} 20: 3, \mathrm{C} 20: 4, \mathrm{C} 22: 4$, and C22:5n-6.

${ }^{6} \mathrm{n}-3=\mathrm{C} 18: 3, \mathrm{C} 20: 5, \mathrm{C} 22: 5 \mathrm{n}-3$, and C22:6.

\section{Cellular Response to Phytohemagglutinin-P}

On d 37 after the initiation of treatments, $0.1 \mathrm{~mL}$ of $1.5 \mathrm{mg}$ of phytohemagglutinin-P (PHA-P)/mL of sterile, isotonic saline was injected intradermally in the ears of each calf. Sterility of the injections was verified by plating $75 \mu \mathrm{L}$ of the working solution on blood agar before and after drawing up the syringes on blood agar. Ear thickness at the site of injection was determined at $0,4,8,12,15,24,32$, and $48 \mathrm{~h}$ postinjection using a pressure-sensitive, digital caliper (Mitutoyo, Kawasaki, Kanagawa, Japan). Results are reported as the change in ear swelling over baseline.

\section{Statistical Analyses}

All repeated data were analyzed by restricted maximum likelihood ANOVA for a completely randomized design using the MIXED procedure of SAS (SAS version 9.1, SAS Institute Inc., Cary, NC). A linear, mixed model with the fixed effects of treatment, time, and the interaction of treatment by time were fitted. The mean model was run with all available covariance structures for the within-subject measurements. The appropriate covariance structure was chosen for each analysis based on the Schwarz's Bayesian information criterion. Baseline measurements were used as covariates during the analyses. Means separation was performed at each time for significant treatment $\times$ time interactions using a sliced effect multiple comparison approach with a Tukey-Kramer adjustment (SAS version 9.1, SAS Institute Inc.). Fecal scores were averaged by week, and the data were normally distributed and therefore analyzed as a continuous repeated measure. Nonrepeated data were analyzed by least squares ANOVA using the GLM procedure of SAS (SAS version 9.1, SAS Institute Inc.).

Repeated data were tested for normality of the residuals by evaluating the Shapiro-Wilk statistic, normal probability plots of the residuals, and histograms of the residuals using the UNIVARIATE procedure of SAS (SAS version 9.1, SAS Institute Inc.). Data that were not normally distributed were transformed and normalized before statistical analyses. In addition, all nonrepeated data were evaluated for homogeneity of variance. Least squares means $( \pm \mathrm{SEM})$ are reported throughout. Orthogonal comparisons were performed to determine the linear and quadratic effects of FO in milk replacer. Treatment differences with $P \leq 0.05$ were considered significant, and $0.05>P \leq 0.10$ were considered tendencies.

\section{RESULTS}

\section{Diets and Calves}

Chemical composition of the 3 milk replacers was not different except for the expected differences in FA composition (Table 1). Adding FO to milk replacer increased the percentage of arachidonic acid (C20:4, n-6), $\mathrm{EPA}$, and DHA. Inclusion of FO decreased the total n-6 FA and increased the total n-3 FA composition; consequently, the n-6:n-3 FA ratio was significantly decreased in the FO-supplemented milk replacers.

During the course of the study, 13 calves died, 4 in both the control and blend and 5 in the fish treatment. Thirty-eight calves, 13 in both the control and blend and 12 in the fish treatment were available for statisti- 


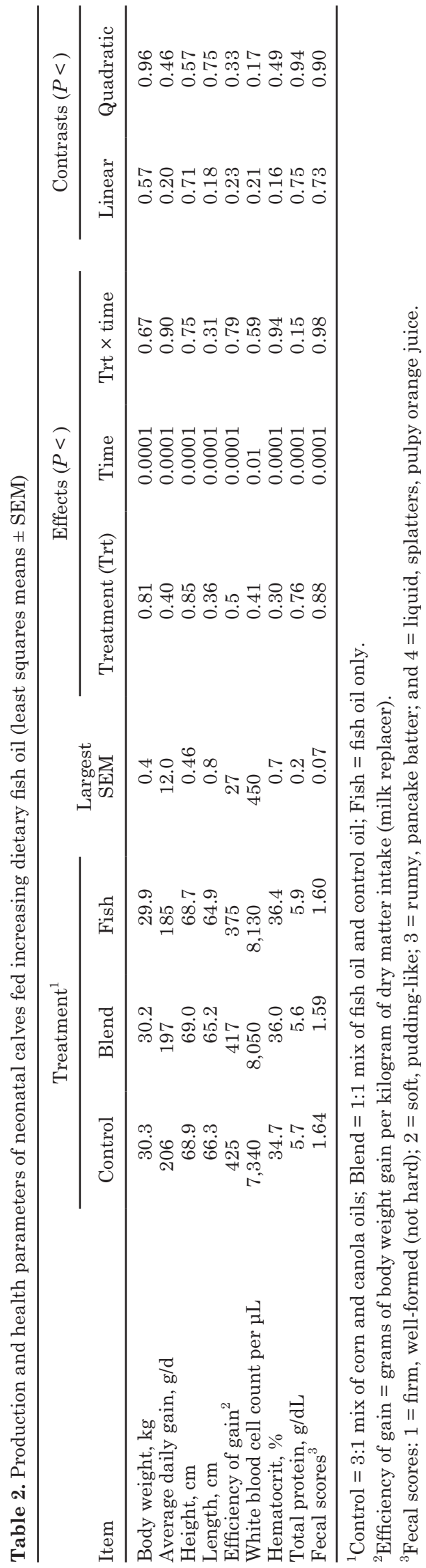

cal analyses. During the first week of the study, there was an attaching and effacing $E$. coli outbreak, which accounted for the high mortality. However, by the second week, the outbreak was controlled.

\section{Production Performance and Health}

There were no treatment effects on growth measurements including BW, wither height, and body length (Table 2). Average daily gain, efficiencies of gain, fecal score, white blood cells, hematocrit, and total protein were also not different among treatments. There were time effects on all production and health parameters measured. The weekly average daily gains are shown in Figure 1. All production measurements followed similar growth patterns to average daily gain. Hematocrit, white blood cells, and total blood protein decreased with maturity. In addition, fecal scores decreased with age (Figure 2). There were no changes in respiratory score within or among treatments throughout the study (data not shown). Finally, there were no differences among treatments on the mean number of days calves needed oral fluid rehydration $(4.4,4.6,4.6 \pm 0.63 \mathrm{~d})$ for the control, blend, and fish treatments, respectively.

\section{Effect of FO on Phagocytosis and Oxidative Burst}

Monocytes and PMN displayed similar phagocytic kinetics. For both cell types, there was a dramatic increase in both the percentage of cells phagocytosing and the mean fluorescence intensity within the 2 -min incubation with $E$. coli. In addition, both measures of phagocytosis were further increased at the 15-min incubation. The effects of treatment on the ability of PMN and monocytes to actively phagocytose $E$. coli are shown in Figure 3A to D. There were no effects of treatment or the interaction of treatment and time on the ability of PMN to phagocytose. However, there was a treatment $\times$ time interaction $(P<0.05)$ on the percentage of monocytes that actively phagocytosed, and both the blend and fish treatments had decreased proportions of phagocytosing monocytes compared with the control after the 2-min incubation, but after the 15-min incubation, they had greater proportions than the control. The mean fluorescence intensity followed a similar pattern, and a tendency $(P<0.08)$ for a treatment $\times$ time interaction was observed.

There was a quadratic effect $(P<0.05)$ for the percentage of PMN producing an oxidative burst (Figure 4). However, there were no differences on the mean fluorescence intensity between treatments.

\section{Effect of FO on the Humoral Response}

There was an interaction between treatment and time $(P<0.03)$ on the production of OVA-specific IgG 


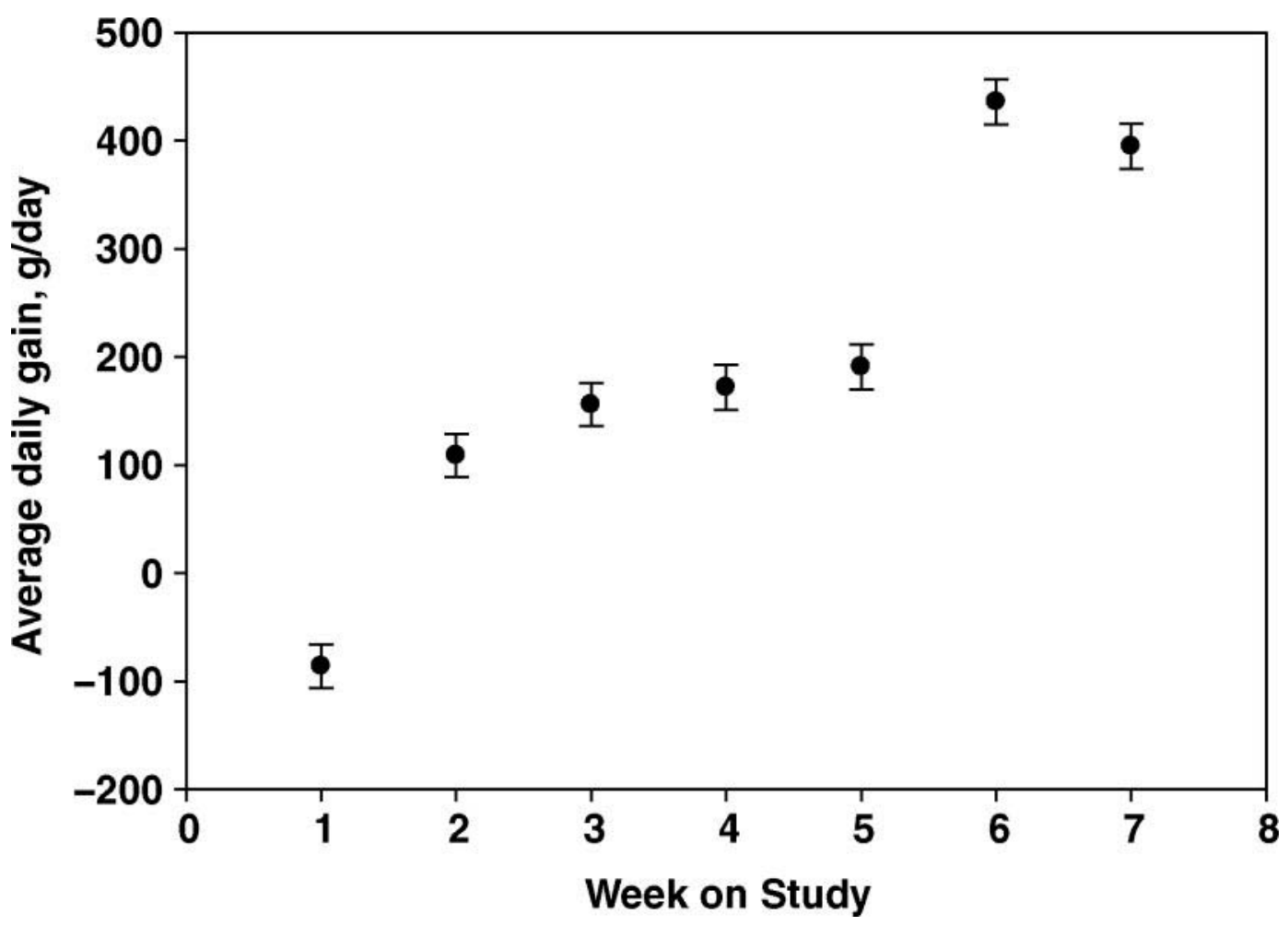

Figure 1. Weekly daily gains averaged over all treatments. There was a time effect $(P<0.001)$.

(Figure 5). Although no effect of treatment was evident on the primary response, the blend treatment had an attenuated secondary response. The vaccination protocol used in the current study unfortunately did not induce a quantifiable IgM response in the primary response.

\section{Effect of FO on Cutaneous Hypersensitivity Reaction}

There was an interaction of treatment and time $(P$ $<0.03$ ) on the change in ear thickness after an intradermal injection with PHA-P (Figure 6). Calves fed the blend treatment had delayed ear swelling but reached responses similar to other treatments by $8 \mathrm{~h}$ after the injection of PHA-P.

\section{Weekly Biochemical Analyses}

A treatment $\times$ time interaction $(P<0.03)$ on serum glucose concentrations (Figure 7A) was evident. Glucose concentrations decreased over the first $14 \mathrm{~d}$ but increased by $21 \mathrm{~d}$ after the initiation of treatments; however, sliced effects revealed no treatment difference at any time. Similar to the glucose data, insulin decreased over the first $14 \mathrm{~d}$ but increased at 21 and $42 \mathrm{~d}$ (Figure 7B). Blood urea nitrogen concentrations were greatest on 7 and $14 \mathrm{~d}$ and decreased by 21 and
$42 \mathrm{~d}$ (Figure 7C). No treatment effects were evident on serum BUN concentration. The concentrations of serum NEFA were variable within the first $21 \mathrm{~d}$ (Figure $7 D)$, and there was an interaction of treatment by time $(P<0.02)$. Sliced effects revealed that at only d 21 , there was a treatment difference $(P<0.001)$, whereas FO calves had decreased NEFA compared with control and blend calves. There were no differences in serum NEFA at $42 \mathrm{~d}$. Serum TG concentrations remained relatively constant for the first $21 \mathrm{~d}$; however, a treatment $\times$ time interaction was apparent $(P<0.05)$, whereas the FO calves had decreased TG concentrations at 21 d compared with control and blend calves (Figure 7E). Finally, serum leptin decreased over the first $14 \mathrm{~d}$, but concentrations recovered by $21 \mathrm{~d}$ (Figure 7F). There was no effect of treatment or interaction of treatment and time on either serum insulin or leptin concentrations.

\section{DISCUSSION}

The effects of supplementing milk replacer with FO on a range of immunological measures representative of key functional responses as well as the overall production and health of calves were investigated. Supplementing milk replacer with FO dramatically altered the FA composition of plasma and peripheral blood 


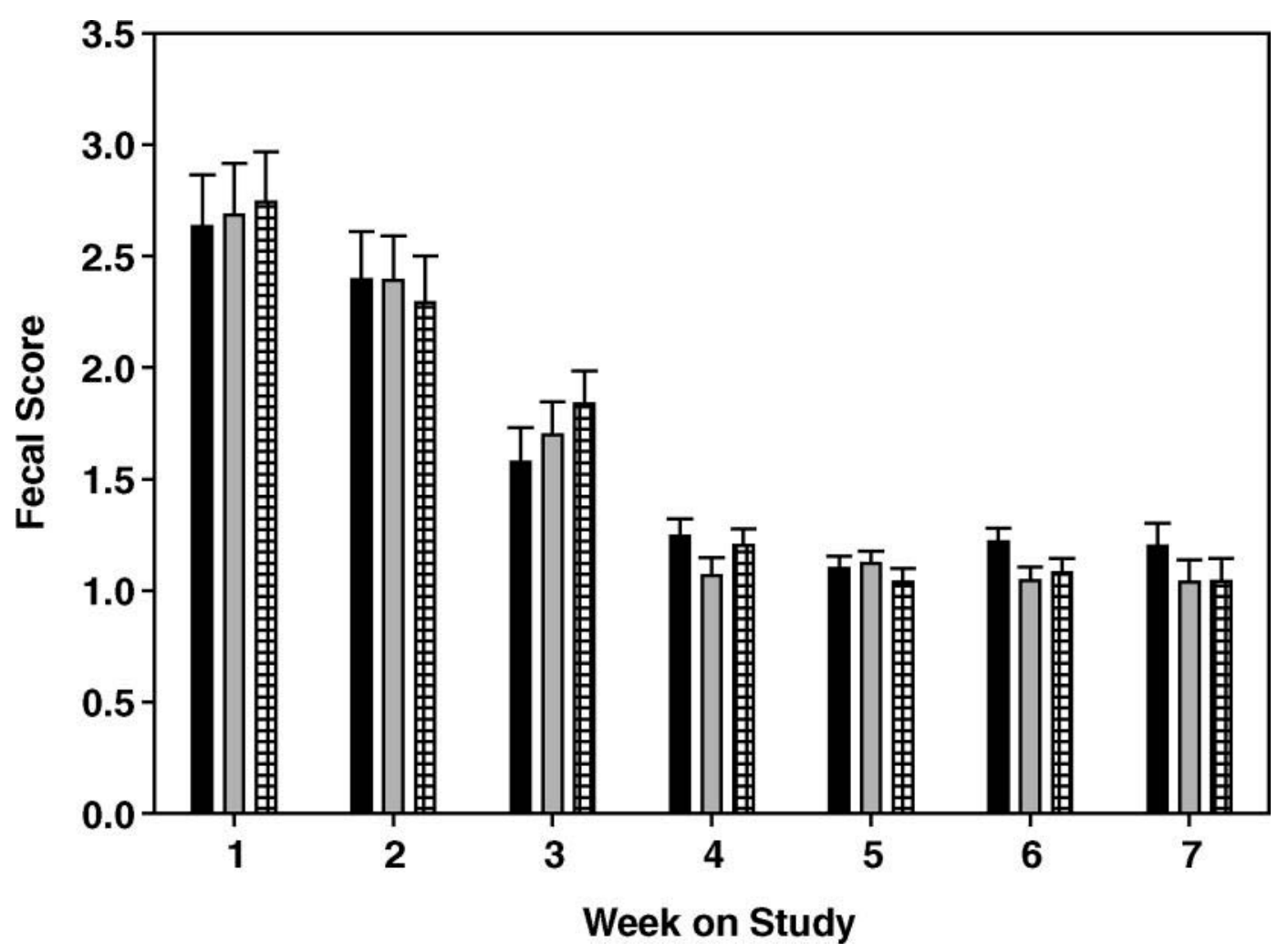

Figure 2. Weekly fecal scores where 1 = firm, well-formed (not hard); 2 = soft, pudding-like; $3=$ runny, pancake batter; and $4=$ liquid, splatters, pulpy orange juice of calves. Fecal scores were collected multiple times daily and were averaged by week before analysis. There were no treatment or treatment $\times$ time interaction effects; however, there was a time effect $(P<0.001)$. Control $=$ black bars; Blend $=$ gray bars; Fish $=$ checkered bars.

mononuclear cell (PBMC) phospholipids of calves (our unpublished data). Briefly, in control calves, with the exception of adrenic acid, all polyunsaturated FA decreased with maturity. Supplementing FO increased the incorporation of all n-3 FA, and the effect was linear with respect to FO dose. Many n-6 FA were decreased in calves supplemented with FO. Therefore, the ratio of n-6:n-3 dramatically decreased in FO-fed calves.

In the present study, growth rates were not significantly different among treatments. Furthermore, there were no treatment differences in the efficiency of BW gain. The only other study that we are aware of that involved feeding FO to dairy calves, feeding high concentrations of FO, 16.6 and $33.2 \%$ of the lipid as FO, did not affect calf performance (Jenkins and Kramer, 1990). The level of FO supplementation in the present study did not influence indices of health. However, feeding high concentrations of FO, $17 \mathrm{~g} / 100$ $\mathrm{g}$ of diet per day and $1.25 \mathrm{~g}$ of EPA + DHA kg/BW per day, decreased the clearance of influenza virus from the lungs of mice (Byleveld et al., 1999) and Staphylococcus aureus from the lungs of neonatal rabbits (D'Ambola et al., 1991), respectively. On the other hand, moderate levels of FO, $55 \mathrm{mg}$ of EPA + DHA/ $\mathrm{kg}$ of BW per day, had no effect on lung staphylococcal clearance (D'Ambola et al., 1991). In rats, replacing either 20 or $40 \%$ of the FA in a parenteral infusate containing $5 \mathrm{~g}$ of total $\mathrm{FA} / 100 \mathrm{~mL}$ of total infusate with FA from FO normalized splanchnic blood flow and decreased the number of viable bacteria in mesenteric lymph nodes and liver after a 24 -h intravenous infusion of endotoxin (Pscheidl et al., 2000). The decreased bacterial counts observed in the FO treatments were attributed to an increased killing of translocated bacteria and not due to a reduction in the translocation rate. Taken together, these data suggest that feeding high concentrations of FO impaired the ability of the immune system to control an infection; however, more moderate levels had no effects. Furthermore, during the clinical course of endotoxemia, increased consumption of FO improved immune function and subsequent recovery from disease. Data from the current study indicate that supplementing calves with FA from FO at a dose of either 5 or $10 \%$ of the total lipid did not influence overall health. More information on the ability of calves supplemented over a wider range of FO intakes to respond to various naturally occurring and experimentally induced infections is warranted. 
A

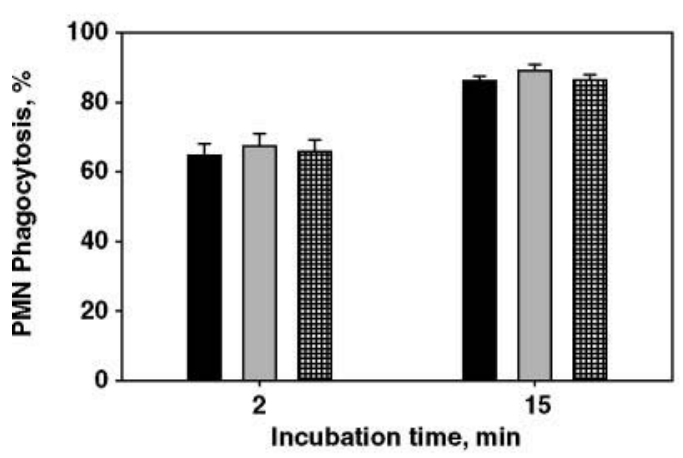

C

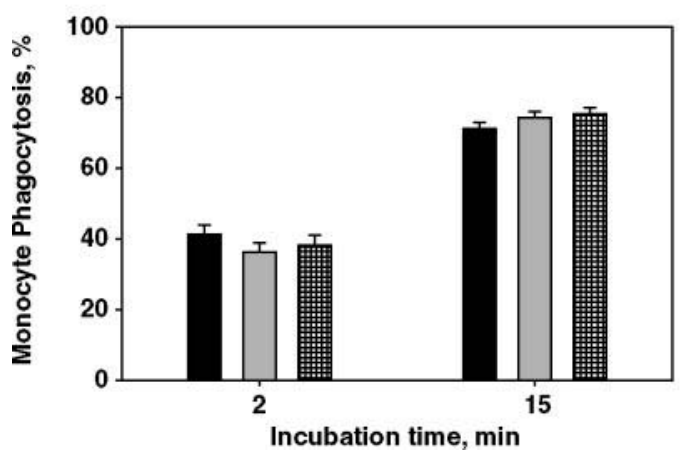

B

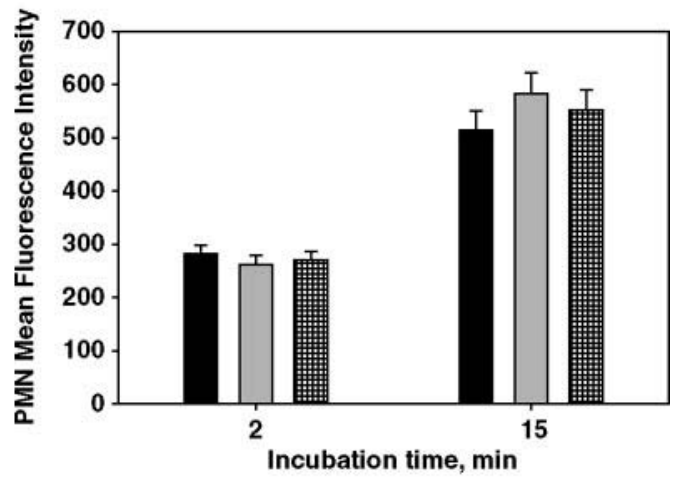

D

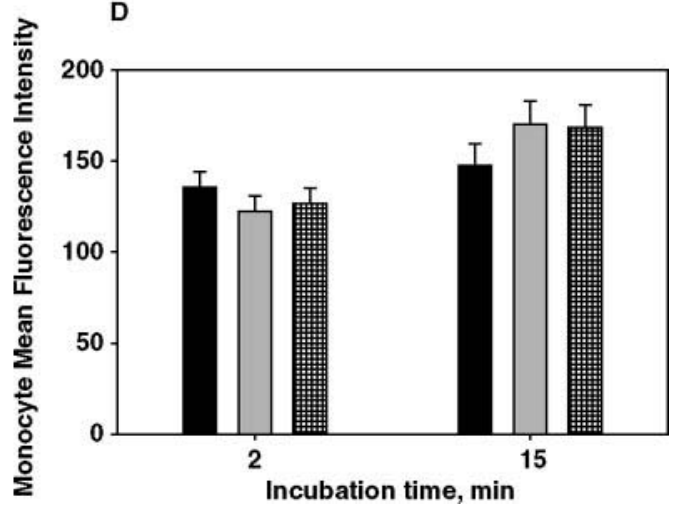

Figure 3. (A) Percentages of polymorphonuclear neutrophils (PMN) phagocytosing fluorescein isothiocyanate (FITC)-labeled Escherichia coli. There were treatment or treatment $\times$ time interactions on the ability of PMN to phagocytose. (B) Mean fluorescence intensity of the FL-1 channel from PMN phagocytosing FITC-labeled $E$. coli. There were treatment or treatment $\times$ time interactions on the mean intensity of PMN phagocytosis. (C) Percentages of monocytes phagocytosing FITC-labeled E. coli. A treatment $\times$ time interaction $(P<0.05)$ on the percentage of monocytes actively phagocytosing was observed. (D) Mean fluorescence intensity of the FL-1 channel from monocytes phagocytosing FITClabeled E. coli. The interaction between treatment and time for the mean fluorescence intensity of monocyte phagocytosis reached a tendency $(P<0.08)$. Control = black bars; Blend = gray bars; Fish $=$ checkered bars.

Phagocytosis is one of the initial and most important defenses in the control of bacterial and fungal infections. In the present study, we investigated the kinetic responses of both PMN and monocytes to phagocytose a preopsonized $E$. coli. There were no treatment differences for either the rate or maximal phagocytic capacities of PMN. Uptake and incorporation of unsaturated FA rather than saturated FA into tissue-cultured macrophages increased the ability of those macrophages to phagocytose unopsonized zymosan particles (Avery et al., 1995). Although phagocytosis was related to the unsaturated FA composition of macrophages, increased EPA and DHA had lesser effects relative to macrophages rich in linoleic, a-linolenic, and arachidonic acids (Calder et al., 1990). Therefore, the net effect of supplementing polyunsaturated FA on phagocytosis cannot be solely attributed to changes in membrane fluidity. The lack of an effect seen in the present study might be due to an apparent balance between membrane fluidity and the incorporation of EPA and DHA into immune cell phospholipids. Despite the lack of an effect on
PMN, a treatment $\times$ time interaction on the phagocytic capacities of monocytes was evident. However, when the data were sliced by time, there were no significant mean differences at either time. The small changes in monocyte phagocytosis likely would not influence host defense.

The production of reactive oxygen species by NADPH oxidase of PMN is a critical mechanism to kill phagocytosed bacteria. In the present study, there was a significant quadratic relationship between the amount of FO in the diet and the proportion of PMN positive for producing an oxidative burst. However, there was no difference among treatments in the mean fluorescence intensity of those cells (Figure 3D). Supplementing EPA at either a low $(1.35 \mathrm{~g} / \mathrm{d})$, moderate $(2.7 \mathrm{~g} / \mathrm{d})$, or high $(4.05 \mathrm{~g} / \mathrm{d})$ dose suppressed the PMN oxidative burst in elderly male subjects (Rees et al., 2006); however, no effect was evident in young men. In contrast, a negative correlation was evident between the oxidative burst of PMN and the n-6:n-3 FA ratio of PBMC (Kew et al., 2003). In addition, a positive cor- 


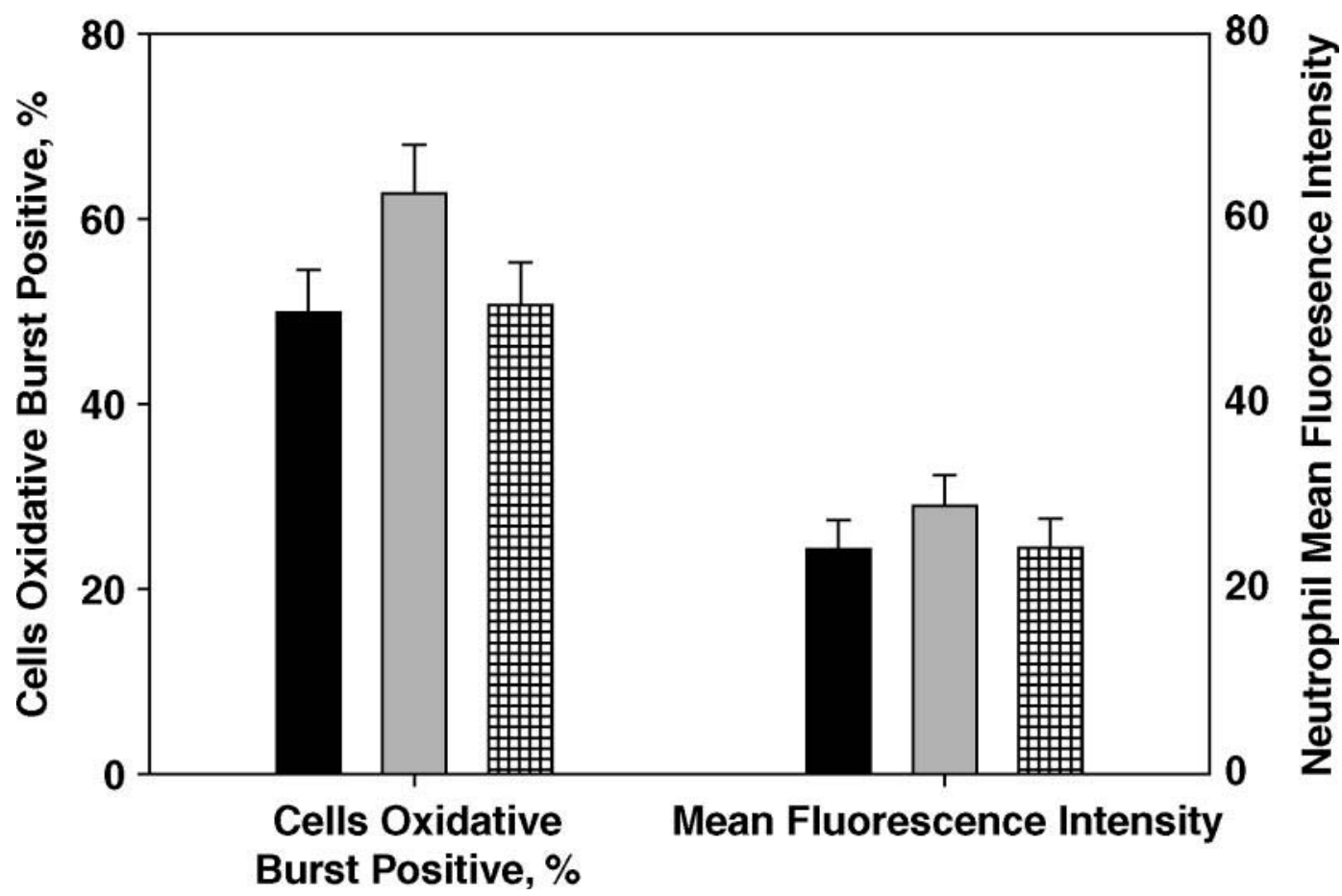

Figure 4. Percentages of polymorphonuclear neutrophils producing an oxidative burst and the mean fluorescence intensity of the FL-1 channel after incubation with fluorescein isothiocyanate-labeled Escherichia coli. There was a quadratic effect $(P<0.05)$ of supplemental fish oil on the percentage of polymorphonuclear neutrophils positive for an oxidative burst; however, no effect was evident for the mean fluorescence intensity. Control = black bars; Blend = gray bars; Fish = checkered bars .

relation existed between oxidative burst and the DHA content of PBMC, but not for EPA. The apparent contradiction between these studies might be explained by the different methodological approaches. Kew et al. (2003) studied variation in habitual intakes of FA and its relationship to immune functions, whereas the other studies supplemented high concentrations of FO or EPA only. Further studies will need to define the dose response relationship, the effect of age, and differentiate between the effects of EPA and DHA and the balance between each.

There was no effect of treatment on the primary humoral response to OVA + Quil A (Figure 5). Therefore, the concentrations of dietary FO used did not affect antigen recognition, presentation, or the activation of both naïve T and B lymphocytes. This observation agrees with previous work in which supplementing diets with FO had no influence on the primary humoral response (Virella et al., 1991). In contrast, chickens fed $7 \%$ FO for 3 wk had increased titers against sheep red blood cells $7 \mathrm{~d}$ after a primary vaccination (Fritsche et al., 1991).

Despite the lack of a treatment effect on the primary humoral response, a quadratic effect was evident on the secondary humoral response, whereas blend had an attenuated response (Figure 5). Feeding marked in- creases of FO, greater than $3 \mathrm{~g} / 100 \mathrm{~g}$ of diet per day or n-6:n-3 FA of the diet less than 1.4:1, had either no effect (Hall et al., 2003) or increased (Selvaraj and Cherian, 2004) the secondary humoral response. In contrast, 3 of 4 healthy human volunteers that received EPA at a dose of $2.4 \mathrm{~g} / \mathrm{d}$ for $6 \mathrm{wk}$ had longitudinal decreases in total serum Ig (Virella et al., 1991). Taken together, the relationship between dietary FO or EPA and DHA in the diet and the secondary humoral response may be quadratic. Supporting this theory, in a study by Wander et al. (1997), although not significant, a numerical quadratic relationship between the n-6:n-3 FA ratio of the diet and the secondary humoral response to keyhole limpet hemocyanin was observed. It is important to note that the variance of the humoral response in that study was substantially greater than the variance in the current study. The precise mechanism for the quadratic relationship is unknown but may include effects on germinal center affinity maturation or Ig class switching. This is an area that warrants further research. The neonatal period is a time when calves are exposed to immunogens for the first time and undergo active immunization; as well, many vaccination protocols begin when calves are still bottle-fed. Any effect on adaptive immunity during this period might manifest later in life. 


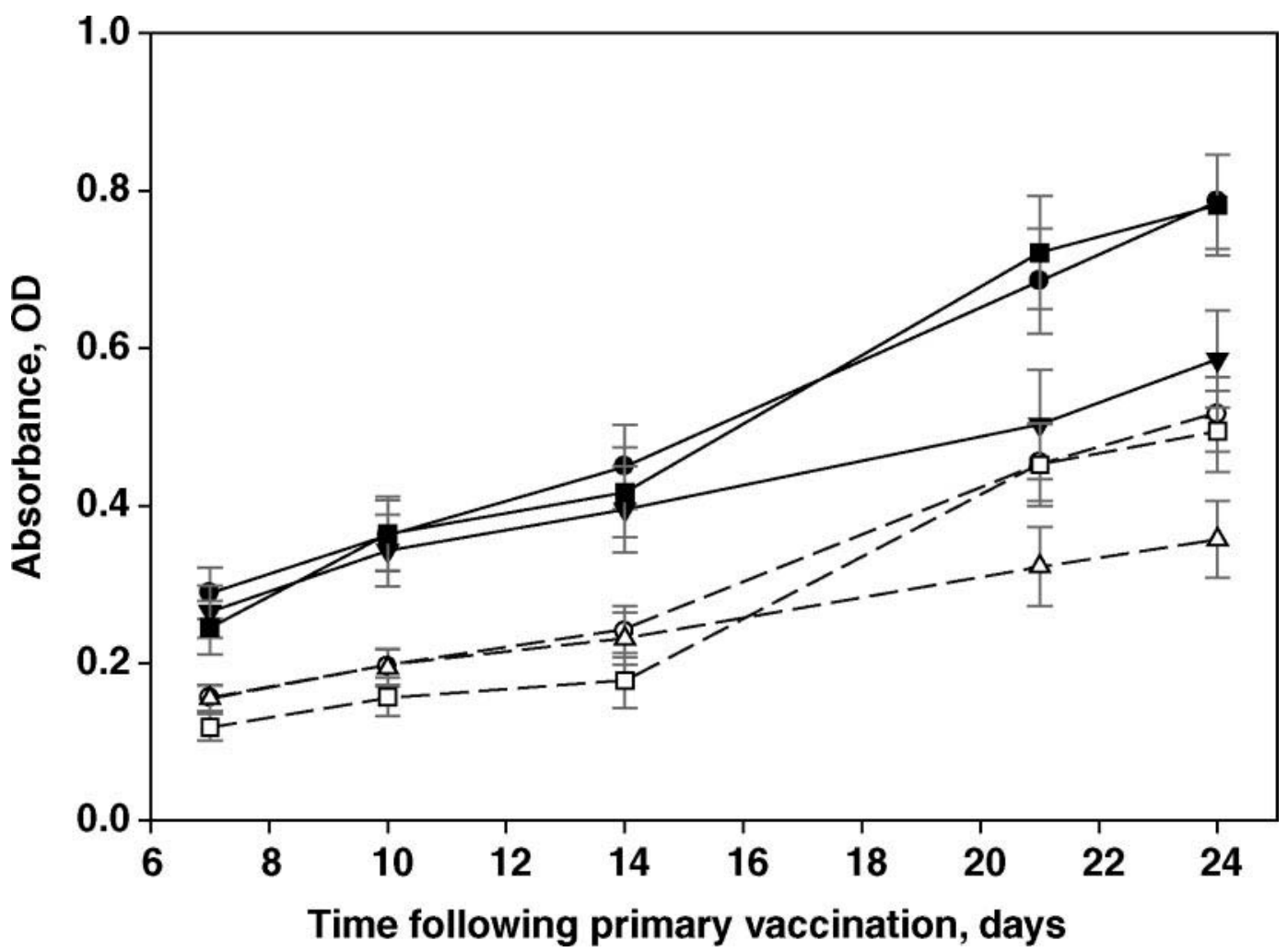

Figure 5. Primary and secondary ovalbumin-specific IgG response to a vaccination with ovalbumin + Quil A. Calves were vaccinated on d 0 and again on d 14 after initiation of treatments. A sandwich ELISA was used to determine ovalbumin-specific IgG. Data are presented relative to a positive control, a pool of nonlactating adult cows that were vaccinated under the same conditions. The positive control was adjusted to 1.0 optical density (OD). An interaction of treatment and time $(P<0.03)$ was evident, whereas there was no effect during the primary response, but blend calves had an attenuated secondary response. Control = circles; Blend $=$ diamonds; Fish $=$ squares. Data are presented at both 50 (solid lines) and 200 (dashed lines)-fold serum dilutions.

The change in skin thickness after an intradermal injection of PHA-P reflects a complex series of events including mitogen-receptor and lymphocyte-macrophage interactions, the release of chemical mediators, changes in vascularity, and cellular proliferation (Lochmiller et al., 1993). The ideal assay to assess cell-mediated immunity is to induce a delayed-type hypersensitivity reaction to a recall antigen rather than using a nonspecific mitogen; however, a recall antigen that will elicit a strong delayed-type hypersensitivity reaction without causing cattle to test positive for Mycobacterium tuberculosis does not currently exist (Hernández et al., 2005). Despite the limitations, histological evaluation of PHA-P injection sites at 24 to $48 \mathrm{~h}$ revealed moderate inflammation with a mixed population of mononuclear cells (Hernández et al., 2005). The attenuated response of blend calves at $4 \mathrm{~h}$ after the injection of PHA-P was likely delayed inflammation and not a decreased mononuclear cell response. Therefore, our results agree with previous studies that demonstrated low to moderate increases in the intake of $\mathrm{n}-3,2.1 \mathrm{~g}$ of EPA $+1.1 \mathrm{~g}$ of $\mathrm{DHA} / \mathrm{d}$, respectively, did not impair mononuclear proliferation (Yaqoob et al., 2000). In contrast, feeding moderate levels of n-3 FA from FO, $1.6 \mathrm{~g}$ of EPA, and $0.72 \mathrm{~g}$ of DHA/d attenuated ex vivo mitogenic lymphocyte proliferation in elderly women (Meydani et al., 1991). Furthermore, others reported that feeding high concentrations of FO, $16 \mathrm{~g}$ of FO/100 g of diet per day or $10 \mathrm{~g}$ of EPA or DHA $/ \mathrm{kg}$ of BW per day to mice decreased cell-mediated immunity, measured by decreased ex vivo lymphocyte proliferation, in vivo delayed-type hypersensitivity, and graft versus host responses (Calder, 1996).

a-Tocopherol increased lymphocyte proliferation in response to mitogens (Lee and Wan, 2000). Furthermore, supplementing adult men with $15 \mathrm{~g}$ of a concentrated n-3 FA FO decreased lymphocyte proliferation; however, when the men were supplemented with an additional $200 \mathrm{mg}$ of a-tocopherol, the inhibition of lymphocyte proliferation was no longer apparent (Kramer et al., 1991). Calves in this study were supplemented with 3 times the current minimum recommendations for vitamin $\mathrm{E}$; therefore, the lack of an effect of $\mathrm{FO}$ on cell-mediated immunity may be due to the counter- 


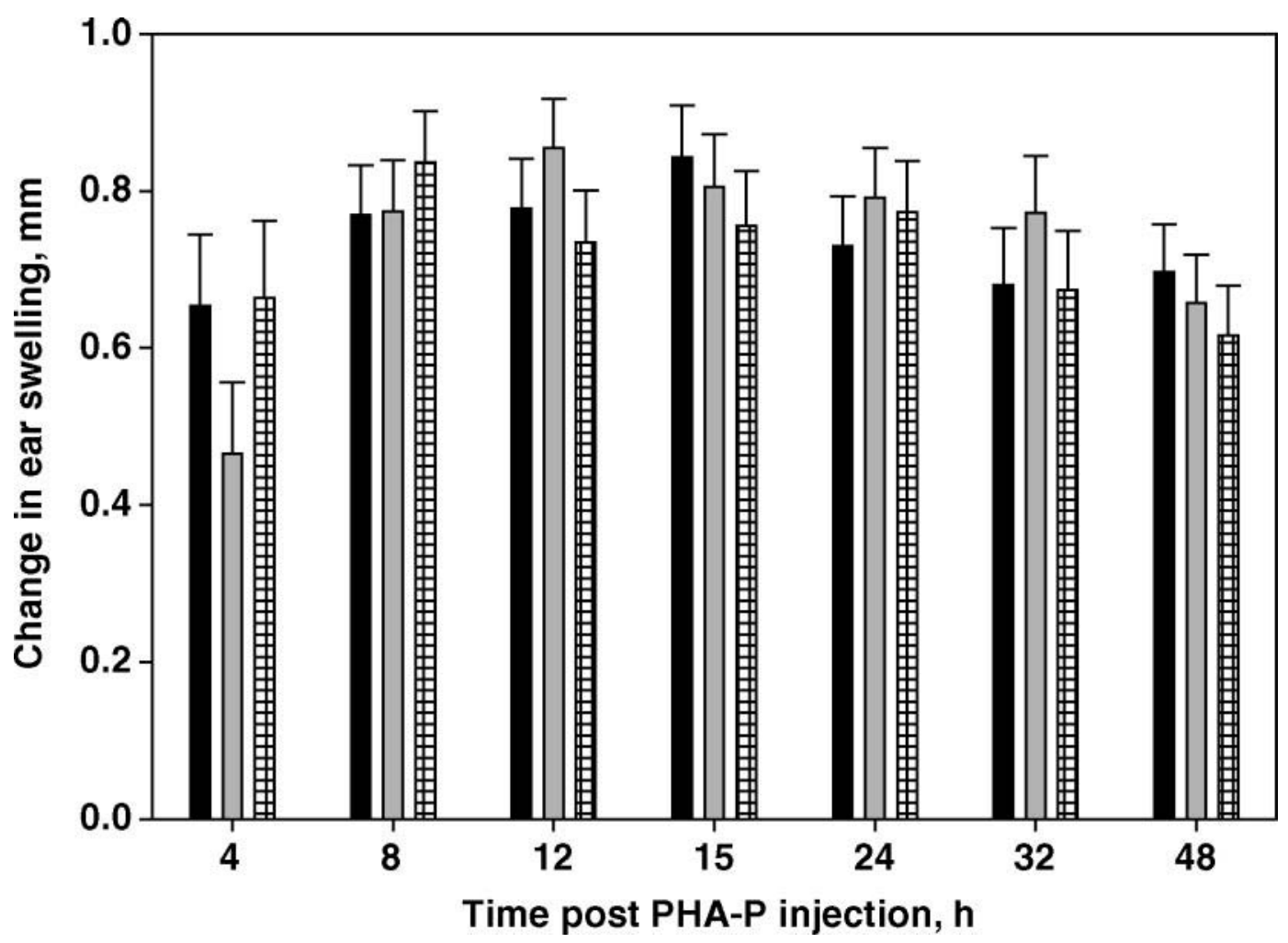

Figure 6. Cutaneous hypersensitivity reaction to an intradermal injection with phytohemagglutinin-P (PHA-P). Data are reported as the change in ear thickness over baseline. There was a treatment $\times$ time interaction $(P<0.03)$ on ear swelling, whereas, the blend treatment had an attenuated response at $4 \mathrm{~h}$ but reached similar levels as both the control and fish treatments at $8 \mathrm{~h}$. Control $=$ black bars; Blend $=$ gray bars; Fish $=$ checkered bars.

regulatory effects of vitamin E. Furthermore, many factors influence the effects of FO on mononuclear cell function, including age, sex, habitual diet, the quantity of n-3 FA in the total diet, and the concentration of other nutrients including a-tocopherol.

Supplementing FO in milk replacer had treatment $x$ time interactions on serum concentrations of glucose, NEFA, and TG. A clear pattern is not apparent, and the interpretation of these interactions is difficult given that these calves were in an unstable condition. The fact that all treatments followed similar temporal patterns may be more biologically significant. The metabolic data from the calves at $42 \mathrm{~d}$ would best reflect the effects of FO on the metabolism of calves, because at this time, all calves were apparently free from any confounding clinical signs of disease. Sliced effects at $42 \mathrm{~d}$ did not reveal any treatment effects.

The metabolic profile that included decreased serum concentrations of glucose, insulin, and leptin and increased serum concentrations of BUN and NEFA of calves during the first 2 wk of the study indicated that the calves were in a catabolic state. Further supporting the catabolic metabolic profile, calves lost BW during the first week of the study, and the increase in
BW during the second week was less than that of the subsequent weeks. During the first $2 \mathrm{wk}$, calves were fed for average daily gains of $200 \mathrm{~g} / \mathrm{d}$, whereas during wk 3 to 5, calves were fed for gains of 400 and $600 \mathrm{~g} / \mathrm{d}$ thereafter. The altered metabolic status during early life was multifactorial and likely included adaptation of the enterocyte to nutrients, decreased absorptive capacity of the enterocyte due to damage by microbial toxins, increased maintenance requirements due to an activated innate immune response, or Jersey nutrient requirements are underestimated. Supporting the last statement, the Jersey calves did not gain as predicted throughout the study (Figure 1).

\section{CONCLUSIONS}

Supplementing milk replacer with moderate levels of n-3 FA from FO influenced the phagocytic function of monocytes, oxidative burst capacity of neutrophils, and the secondary humoral response of Jersey calves. Level of FO supplemented in the formula was critical to the response in these immunological parameters. The current study reinforced that n-3 FA affect specific immune responses at many mechanistic levels, and the 

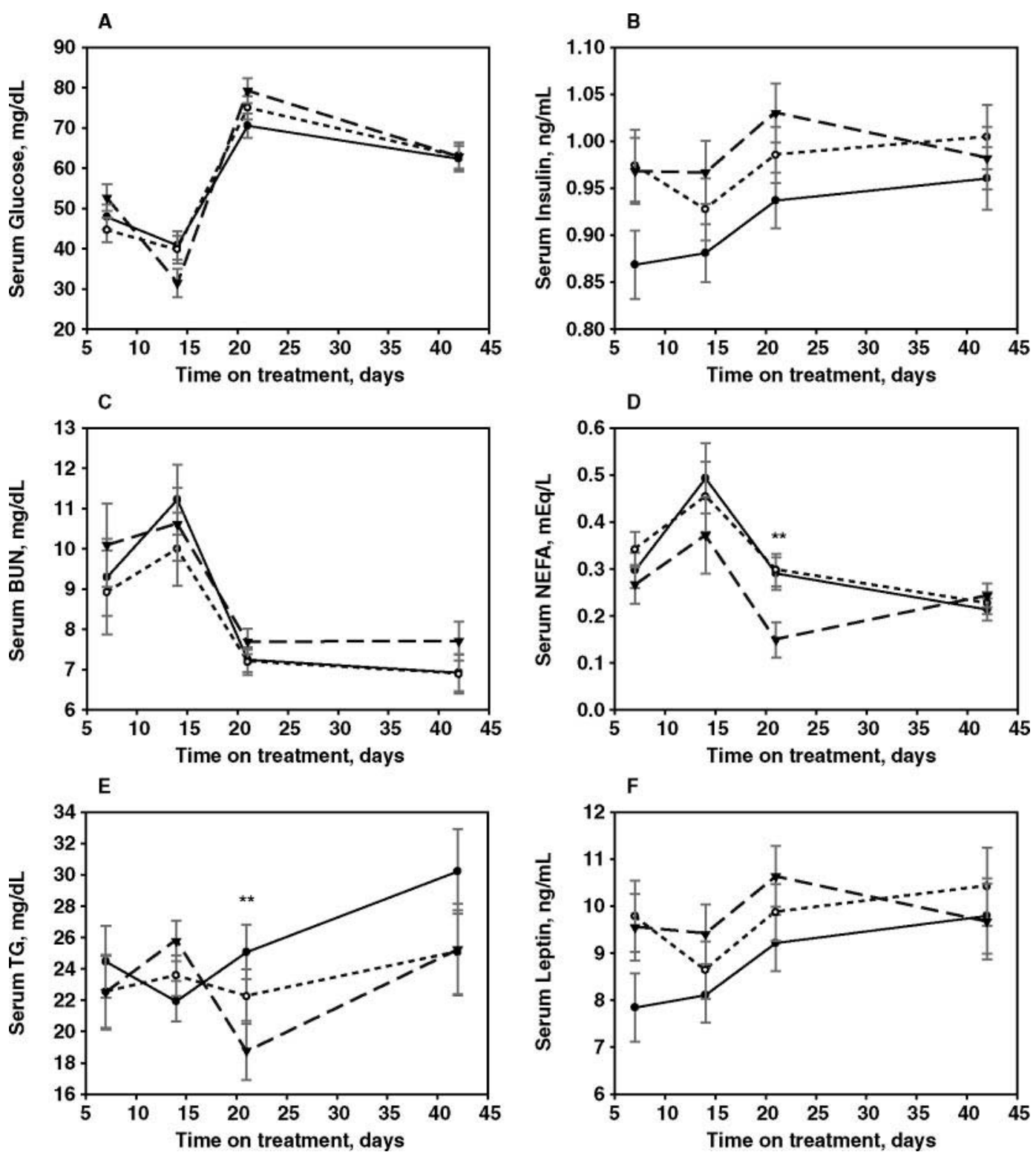

Figure 7. (A) Effects of supplemental fish oil on the weekly blood glucose concentrations of neonatal calves. A treatment $\times$ time interaction $(P<0.03)$ was evident. (B) Effects of supplemental fish oil on the weekly serum insulin concentrations of neonatal calves. No effects of treatment were evident. (C) Effects of supplemental fish oil on the weekly serum BUN concentrations of neonatal calves. No effects of treatment were evident. (D) Effects of supplemental fish oil on the weekly serum NEFA concentrations of neonatal calves. A treatment $\times$ time interaction $(P<0.02)$ was evident. Sliced time effects noted as $* P<0.10$ and $* * P<0.05$. (E) Effects of supplemental fish oil on the weekly serum triacylglycerol (TG) concentrations of neonatal calves. A treatment $\times$ time interaction $(P<0.05)$ was evident. Sliced time effects noted as $* P<0.10$ and ${ }^{* *} P<0.05$. (F) Effects of supplemental fish oil on the weekly serum leptin concentrations of neonatal calves. $\bullet=$ Control; $\circ$ $=$ Blend; $\boldsymbol{\nabla}=$ Fish. No effects of treatment were evident. Error bars represent \pm SEM.

net effect was likely an intricate balance between those mechanisms. However, in the current study, replacing 5 to $10 \%$ of the lipid as FA from FO did not influence either production performance or overall measures of health. Future research should focus on defining the dose response relationships, begin to elucidate the potential mechanisms, as well as use disease challenge models.

\section{ACKNOWLEDGMENTS}

We thank G. D. Cruz and S. O. Juchem for their assistance in collecting the data and caring for the calves; S. J. Taylor, T. Graham (Veterinary Consulting Service, Davis, CA), J. T. Stott, and M. Blanchard for their technical support, and J. E. Santos and K. C. Klasing for their comments on the manuscript. Additionally, we 
acknowledge and appreciate W. Pitroff and D. Keisler for measuring insulin and leptin. Finally, we acknowledge the contributions of the American Jersey Cattle Association (Reynoldsburg, OH), Omega Proteins Inc. (Houston, TX), Calva Products (Acampo, CA), Western Milling (Goshen, CA), Mendes Calf Ranch (Tipton, CA), Hilmar Cheese Company (Hilmar CA), Tech Mix (Stewart, MN), and Pfizer Animal Health (St. Louis, $\mathrm{MO})$. Special thanks to the dairy producers who donated the Jersey calves.

\section{REFERENCES}

Avery, S. V., D. Lloyd, and J. L. Harwood. 1995. Temperaturedependent changes in plasma-membrane lipid order and the phagocytic activity of the amoeba Acanthamoeba castellanii are closely correlated. Biochem. J. 312:811-816.

Ballou, M. A., G. D. Cruz, W. Pitroff, D. H. Keisler, and E. J. DePeters. 2008. Modifying the acute phase response of Jersey calves by supplementing milk replacer with omega-3 fatty acids from fish oil. J. Dairy Sci. 91:3478-3487.

Byleveld, P. M., G. T. Pang, R. L. Clancy, and D. C. Roberts. 1999. Fish oil feeding delays influenza virus clearance and impairs production of interferon-Y and virus-specific immunoglobulin A in the lungs of mice. J. Nutr. 129:329-335.

Calder, P. C. 1996. Immunomodulatory and anti-inflammatory effects of n-3 polyunsaturated fatty acids. Proc. Nutr. Soc. 55:737-774.

Calder, P. C. 2006. n-3 Polyunsaturated fatty acids, inflammation, and inflammatory diseases. Am. J. Clin. Nutr. 83(Suppl.):1505S$1519 \mathrm{~S}$.

Calder, P. C., J. A. Bond, D. J. Harvey, S. Gordon, and E. A. Newsholme. 1990. Uptake and incorporation of saturated and unsaturated fatty acids into macrophage lipids and their effect upon macrophage adhesion and phagocytosis. Biochem. J. 269:807-814.

D’Ambola, J. B., E. E Aeberhard, N. Trang, S. Gaffar, C. T. Barrett, and M. P. Sherman. 1991. Effect of dietary (n-3) and (n-6) fatty acids on in vivo pulmonary bacterial clearance by neonatal rabbits. J. Nutr. 121:1262-1269.

Fritsche, K. L., N. A. Cassity, and S. C. Huang. 1991. Effect of dietary fat source on antibody production and lymphocyte proliferation in chickens. Poult. Sci. 70:611-617.

Hall, J. A., K. A. Tooley, J. L. Gradin, D. E. Jewell, and R. C. Wander. 2003. Effects of dietary n-6 and n-3 fatty acids and vitamin $\mathrm{E}$ on the immune response of healthy geriatric dogs. Am. J. Vet. Res. 64:762-772.

Hernández, A., J. A. Yager, B. N. Wilkie, K. E. Leslie, and B. A. Mallard. 2005. Evaluation of bovine cutaneous delayed-type hypersensitivity to various test antigens and a mitogen using several adjuvants. Vet. Immunol. Immunopathol. 104:45-58.

Jenkins, K. J., and J. K. G. Kramer. 1990. Effects of dietary corn oil and fish oil concentrate on lipid composition of calf tissues. J. Dairy Sci. 73:2940-2951.

Kampen, A. H., T. Tollersrud, S. Larsen, J. A. Roth, D. E. Frank, and A. Lund. 2004. Repeatability of flow cytometric and classical measurement of phagocytosis and respiratory burst in bovine polymorphonuclear leukocytes. Vet. Immunol. Immunopathol. 97:105-114.

Kew, S., T. Banerjee, A. M. Minihane, Y. E. Finnegan, C. M. Williams, and P. C. Calder. 2003. Relation between the fatty acid composition of peripheral blood mononuclear cells and measures of immune cell function in healthy, free-living subjects aged 2572 y. Am. J. Clin. Nutr. 77:1278-1286.

Kramer, T. R., N. Schoene, L. W. Douglass, J. T. Judd, R. BallardBarbash, P. R. Taylor, H. N. Bhagavan, and P. P. Nair. 1991. Increased vitamin $\mathrm{E}$ intake restores fish-oil-induced suppressed blastogenesis of mitogen-stimulated T lymphocytes. Am. J. Clin. Nutr. 54:896-902.

Larson, L. L., F. G. Owen, J. L. Albright, R. D. Appleman, R. C. Lamb, and L. D. Muller. 1977. Guidelines toward more uniformity in measuring and reporting calf experimental data. J. Dairy Sci 60:989-991.

Lee, C. Y., and J. M. Wan. 2000. Vitamin E supplementation improves cell-mediated immunity and oxidative stress of Asian men and women. J. Nutr. 130:2932-2937.

Lochmiller, R. L., M. R. Vestey, and S. T. McMurry. 1993. Selected immune responses of adult cotton rats (Sigmodon hispidus) to dietary restriction. Comp. Biochem. Physiol. 104:593-599.

Lofstedt, J., I. R. Dohoo, and G. Duizer. 1999. Model to predict septicemia in diarrheic calves. J. Vet. Intern. Med. 2:81-88.

Mallard, B. A., L. C. Wagter, M. J. Ireland, and J. C. M. Dekkers. 1997. Effects of growth hormone, insulin-like growth factor I, and cortisol on periparturient antibody response profiles of dairy cattle. Vet. Immunol. Immunopathol. 60:61-67.

Meydani, S. N., S. Endres, M. M. Woods, B. R. Goldin, C. Soo, A. Morrill-Labrode, C. A. Dinarello, and S. L. Gorbach. 1991. Oral (n-3) fatty acid supplementation suppresses cytokine production and lymphocyte: Comparison between young and older women. J. Nutr. 121:547-555.

NRC. 2001. Nutrient Requirements for Dairy Cattle. 7th rev. ed. Natl. Acad. Sci., Washington, DC.

Pscheidl, E., M. Schywalsky, K. Tschaikowsky, and T. BökePröls. 2000. Fish oil-supplemented parenteral diets normalize splanchnic blood flow and improve killing of translocated bacteria in a low-dose endotoxin rat model. Crit. Care Med. 28:14891496.

Rees, D., E. A. Miles, T. Banerjee, S. J. Wells, C. E. Roynette, K. W. J. Wahle, and P. C. Calder. 2006. Dose-related effects of eicosapentaenoic acid on innate immune function in healthy humans: A comparison of young and older men. Am. J. Clin. Nutr. 83:331-342.

Roy, J. H. B. 1990. The Calf. 5th ed. Vol. 1. Management of Health. Butterworth Publishers Inc., Boston, MA.

Selvaraj, R. K., and G. Cherian. 2004. Dietary n-3 fatty acids reduce the delayed hypersensitivity reaction and antibody production more than n- 6 fatty acids in broiler birds. Eur. J. Lipid Sci. Technol. 106:3-10.

Virella, G., K. Fourspring, B. Hyman, R. Haskill-Stroud, L. Long, I. Virella, M. La Via, A. J. Gross, and M. Lopes-Virella. 1991. Immunosuppressive effects of fish oil in normal human volunteers: Correlation with the in vitro effects of eicosapentaenoic acid on human lymphocytes. Clin. Immunol. Immunopathol. 61:161167.

Wander, R. C., J. A. Hall, J. L. Gradin, D. Shi-Hua, and D. E. Jewell. 1997. The ratio of dietary (n-6) to (n-3) fatty acids influences immune system function, eicosanoid metabolism, lipid peroxidation, and vitamin E status in aged dogs. J. Nutr. 127:1198-1205.

Yaqoob, P., H. S. Pala, M. Cortina-Borja, E. A. Newsholme, and P. C. Calder. 2000. Encapsulated fish oil enriched in a-tocopherol alters plasma phospholipid and mononuclear cell fatty acid compositions but not mononuclear cell functions. Eur. J. Clin. Invest. 30:260-274. 